\title{
Comparison of Various Classification Techniques Using Different Data Mining Tools for Diabetes Diagnosis
}

\author{
Rashedur M. Rahman, Farhana Afroz \\ Department of Electrical Engineering and Computer Science, North South University, Dhaka, Bangladesh. \\ Email: rashedur@northsouth.edu,farhana_s1_03@yahoo.com
}

Received December $21^{\text {st }}, 2012$; revised January $20^{\text {th }}, 2013$; accepted January $30^{\text {th }}, 2013$

\begin{abstract}
In the absence of medical diagnosis evidences, it is difficult for the experts to opine about the grade of disease with affirmation. Generally many tests are done that involve clustering or classification of large scale data. However many tests could complicate the main diagnosis process and lead to the difficulty in obtaining the end results, particularly in the case where many tests are performed. This kind of difficulty could be resolved with the aid of machine learning techniques. In this research, we present a comparative study of different classification techniques using three data mining tools named WEKA, TANAGRA and MATLAB. The aim of this paper is to analyze the performance of different classification techniques for a set of large data. A fundamental review on the selected techniques is presented for introduction purpose. The diabetes data with a total instance of 768 and 9 attributes ( 8 for input and 1 for output) will be used to test and justify the differences between the classification methods. Subsequently, the classification technique that has the potential to significantly improve the common or conventional methods will be suggested for use in large scale data, bioinformatics or other general applications.
\end{abstract}

Keywords: Classification; Neural Network; Fuzzy Logic; Decision Tree; Performance Measurement

\section{Introduction}

The aim of this study is to investigate the performance of different classification methods using WEKA, TANAGRA and MATLAB tool on Diabetes Dataset, specifically Pima Indian Diabetes Dataset. A major problem in bioinformatics analysis or medical science is to attain the correct diagnosis of certain important information. For the ultimate diagnosis, generally many tests are done that involve clustering or classification of large scale data. All of these test procedures are said to be necessary in order to reach the ultimate diagnosis. However, on the other hand, too many tests could complicate the main diagnosis process and lead to the difficulty in obtaining the end results, particularly in the case where many tests are performed. This kind of difficulty could be resolved with the aid of machine learning which could be used directly to obtain the end result with the aid of several artificial intelligence techniques. Machine learning covers such a broad range of processes that it is difficult to define it precisely. A dictionary definition includes phrases such as to gain knowledge or understanding of or skill by studying the instruction or experience and modification of a behavioral tendency by experienced zoologists and psychologists study learning in animals and humans [1]. The extraction of important information from a large pile of data and its correlations is often the advantage of using machine learning. New knowledge about tasks is constantly being discovered by humans. There is a constant stream of new events in the world and continuing redesign of Artificial Intelligent systems to conform to new knowledge is impractical but machine learning methods might be able to track much of it [1].

There is a substantial amount of research has been done with machine learning algorithms such as Bayes network, Multilayer Perceptron, Decision tree and pruning like J48graft, C4.5, Single Conjunctive Rule Learner like FLR, JRip and Fuzzy Inference System and Adaptive Neuro-Fuzzy Inference System.

\section{Related Work}

A good number of researches have been reported in literature on diagnosis of different deceases. Sapna and Tamilarasi [2] proposed a technique based on neuropathy diabetics. Nerve disorder is caused by diabetic mellitus. Long term diabetic patients could have diabetic neuropathies very easily. There is fifty $(50 \%)$ percent probability to have such diseases which affect many nerves system of the body. For example, body wall, limbs (which is called as somatic nerves) could be affected. On the other hand, internal organ like heart, stomach, etc., 
are known as automatic nerves. In this paper the risk factors and symptoms of diabetic neuropathy are used to make the fuzzy relation equation. Fuzzy relation equation is linked with the perception of composition of binary relations that means they used Multilayer Perceptron NN using Fuzzy Inference System.

Leonarda and Antonio [3] proposed automatic detection of diabetic symptoms in retinal images by using a multilevel perceptron neural network. The network is trained using algorithms for evaluating the optimal global threshold which can minimize pixel classification errors. System performances are evaluated by means of an adequate index to provide percentage measure in the detection of eye suspect regions based on neuro-fuzzy subsystem.

Radha and Rajagopalan [4] introduced an application of fuzzy logic to diagnosis of diabetes. It describes the fuzzy sets and linguistic variables that contribute to the diagnosis of disease particularly diabetes. As we all know fuzzy logic is a computational paradigm that provides a tool based on mathematics which deals with uncertainty. At the same time this paper also presents a computer-based Fuzzy Logic with maximum and minimum relationship, membership values consisting of the components, specifying fuzzy set frame work. Forty patients' data have been collected to make this relationship more strong.

Jeatrakul and Wong [5] presented a comparison of neural network techniques for binary classification problems. The classification performance obtained by five different types of neural networks, i.e., Back Propagation Neural Network (BPNN), Radial Basis Function Neural Network (RBFNN), General Regression Neural Network (GRNN), Probabilistic Neural Network (PNN), and Complementary Neural Network (CMTNN). The comparison is done based on three benchmark data sets obtained from UCI machine learning repository.

Zhou, Purvis and Kasabov [6] described a general method based on the statistical analysis of training data to select fuzzy membership functions to be used in connection with fuzzy neural networks. The technique is first described and then illustrated by means of two experimental examinations for medical data.

Ten-Ho Lin and Von-Wun Soo [7] proposed alternative pruning method based on the Minimal Description Length (MDL) principle. The MDL principle can be viewed as a tradeoff between theory complexity and data prediction accuracy given the theory. A greedy search algorithm of the minimum description length to prune the fuzzy ARTMAP categories one by one was proposed. The experiments showed that fuzzy ARTMAP pruned with the MDL principle gave better performance with fewer categories created compared to original fuzzy ARTMAP and other machine learning systems. They tested those techniques on a number of benchmark clinical databases such as heart disease, breast cancer and diabetes databases.

Faezeh, Hossien, Ebrahim [8] proposed a fuzzy clustering technique (FACT) which determined the number of appropriate clusters based on the pattern essence. Different experiments for algorithm evaluation were performed which showed a better performance compared to the typical widely used K-means clustering algorithm. Data was taken from the UCI Machine Learning Repository [9].

Santi Waulan et al. [10] proposed a new SSVM for classification problems. It is called Multiple Knot Spline SSVM (MKS-SSVM). To evaluate the effectiveness of their method, they carried out an experiment on Pima Indian diabetes dataset. First, theoretical of MKS-SSVM was presented. Then, application of MKS-SSVM and comparison with SSVM in diabetes disease diagnosis was given. The results of this study showed that the MKS-SSVM was effective to detect diabetes disease diagnosis.

\section{Data Set Description}

The characteristics of the data set used in this research are summarized in following Table 1. The detailed descriptions of the data set are available at UCI repository [9].

The objective of this data set was diagnosis of diabetes of Pima Indians. Based on personal data, such as age, number of times pregnant, and the results of medical examinations, e.g., blood pressure, body mass index, result of glucose tolerance test, etc., it is tried to decide whether a Pima Indian individual was diabetes positive or not.

Pima Indian Diabetes Data (PIDD) set is publicly available from the machine learning database at UCI. All patients represented in this data set are females with at least 21 years old of Pima Indian heritage living near Phoenix, Arizona. The problem posed here is to predict whether a person would test positive given a number of physiological measurements and medical test results. This is a two-class problem with class value 1 being interpreted as "tested positive for diabetes". There are 500

Table 1. Characteristics of data sets.

\begin{tabular}{ccccccc}
\hline Data set & No. of example & Input attributes & Output classes & $\begin{array}{c}\text { Total No. of } \\
\text { attributes }\end{array}$ & Missing attributes status & $\begin{array}{c}\text { Noisy attributes } \\
\text { status }\end{array}$ \\
\hline Pima & 768 & 8 & 2 & 9 & No & No \\
\hline
\end{tabular}


examples of class 1 and 268 of class 2 .

This data set is extracted from a larger database originnally owned by the National Institute of Diabetes and Digestive and Kidney Diseases. The purpose of the study is to investigate the relationship between the diabetes diagnostic result and a list of variables that represent physiological measurements and medical attributes. The data set in the UCI repository contains 768 observations and 9 variables with no missing values reported. However, as some researchers point out, there are a number of impossible values, such as 0 body mass index and 0 plasma glucose. Furthermore, one attribute (2-hour serum insulin) contains almost 50\% impossible values. To keep the sample size reasonably large, this attribute is removed from analysis. There are 236 observations that have at least one impossible value of glucose, blood pressure, triceps skin thickness, and body mass index. There are nine variables, including the binary response variable, in this data set; all other attributes are numericvalued. The attributes are given below:

1) Number of times pregnant

2) Plasma glucose concentration a 2 hours in an oral glucose tolerance test

3) Diastolic blood pressure $(\mathrm{mm} \mathrm{Hg})$

4) Triceps skin fold thickness (mm)

5) 2-hour serum insulin (mu U/ml)

6) Body mass index (weight in $\mathrm{kg} /(\text { height in } \mathrm{m})^{\wedge} 2$ )

7) Diabetes pedigree function

8) Age (years)

9) Class variable ( 0 or 1$)$

\section{Methodology}

We use different classification techniques in this research. Those techniques with running parameters are given below:

\subsection{Multilayer Perceptron}

Multilayer perceptron (MLP) [11] is one of the most commonly used neural network classification algorithms. The architecture used for the MLP during simulations with PIDD dataset consisted of a three layer feed-forward neural network: one input, one hidden, and one output layer. Selected parameters for the model are: learningRate $=0.3 / 0.15 ;$ momentum $=0.2$; randomSeed $=0$; validationThreshold $=20$, Number of Epochs $=500$.

\subsection{BayesNet}

BayesNet [12] learns Bayesian networks under the presumptions: nominal attributes (numeric one are pre-descretized) and no missing values (any such values are replaced globally). There are two different parts for estimating the conditional probability tables of the network. In this study we run BayesNet with the SimpleEstimator and K2 search algorithm without using ADTree. K2 algorithm is a greedy search algorithm that works as follows. Suppose we know the total ordering of the nodes. Initially each node has no parents. The algorithm then incrementally adds the parent whose addition increases most of the score of the resulting structure. When no addition of a single parent can increase the score, it stops adding parents to the node. Since an ordering of the nodes is known beforehand, the search space under this constraint is much smaller than the entire space. And we do not need to check for cycles, since the total ordering guarantees that there is no cycle in the deduced structures. Furthermore, based on some appropriate assumptions, we can choose the parents for each node independently.

\subsection{Naïve Byes}

The Naïve Bayes [12] classifier provides a simple approach, with clear semantics, representing and learning probabilistic knowledge. It is termed naïve because is relies on two important simplifying assumes that the predictive attributes are conditionally independent given the class, and it assumes that no hidden or latent attributes influence the prediction process.

\subsection{J48graft (C4.5 Decision Tree Revision 8)}

Perhaps C4.5 algorithm which was developed by Quinlan [13] is the most popular tree classifier till today. Weka classifier package has its own version of $\mathrm{C} 4.5$ known as J48 or J48graft. For this study, C4.5 classifier used in TANAGRA platform and J48graft classifier used in WEKA platform. J48graft is an optimized implementation of $\mathrm{C} 4.5$ rev. 8. J48graft is experimented is this study with the parameters: confidenceFactor $=0.25$; $\operatorname{minNumObj}=2$; subtreeRaising $=$ True; unpruned $=$ False. C4.5 is experimented in this study with the parameters: Min size of leaves $=5$; Confidence-Level for pessimistic $=0.25$. Final decision tree built from the algorithm is depicted in Figure 1.

\subsection{Fuzzy Lattice Reasoning (FLR)}

The Fuzzy Lattice Reasoning (FLR) classifier is presented for inducing descriptive, decision-making knowledge (rules) in a mathematical lattice data domain including space $R^{N}$. Tunable generalization is possible based on non-linear (sigmoid) positive valuation functions; moreover, the FLR classifier can deal with missing data. Learning is carried out both incrementally and fast by computing disjunctions of join-lattice interval conjunctions, where a join-lattice interval conjunction corresponds to a hyperbox in $R^{N}$. In this study we evaluated FLR classifier in WEKA with the parameters: $R$ hoa $=0.5$; Number of Rules $=2$. 


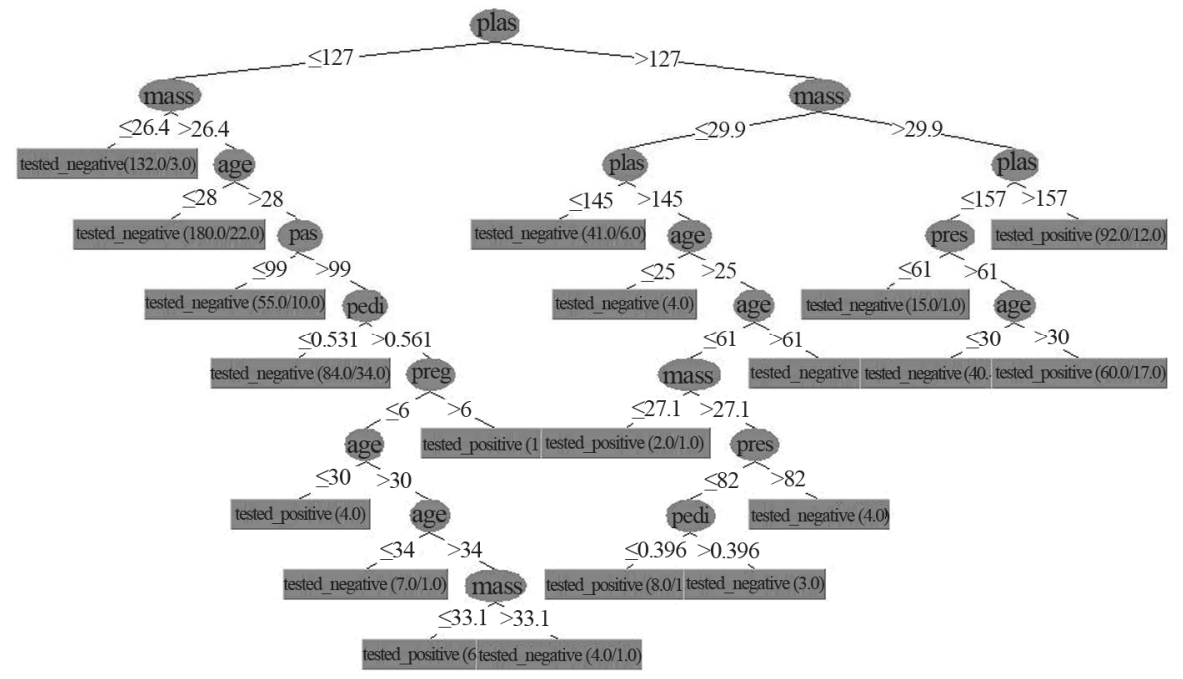

Figure 1. Decision tree for J48graft algorithm for PIDD after experiment with WEKA.

\subsection{JRip (JRipper)}

Repeated Incremental Pruning to Produce Error Reduction (RIPPER) [14] is one of the basic and most popular algorithms. Classes are examined in increasing size and an initial set of rules for the class is generated using incremental reduced-error pruning. In this study, we evaluated RIPPER through JRip, an implementation of RIPPER in WEKA with the parameters: folds $=10$; $\operatorname{minNo}=$ 2 ; optimizations $=2$; seed $=1$; usePruning $=$ true.

\subsection{Fuzzy Inference System (FIS)}

Fuzzy inference system (FIS) is a technology developed for granular rule induction and generalization based on fuzzy logic. Note that since a data cluster can be interpreted as a (fuzzy) granule, data clustering may be closely related to fuzzy rule induction. Neural implementations have provided conventional FIS a capacity for parallel implementation.

\subsection{Adaptive Neuro-Fuzzy Inference System (ANFIS)}

In this work uses ANFIS (Adaptive Neuro-Fuzzy Inference System), a fuzzy classifier that is part of the MATLAB Fuzzy Logic Toolbox [15]. ANFIS (AdaptiveNetwork-Based Fuzzy Inference System) is a fuzzy inference system implemented under the framework of adaptive networks [16]. ANFIS is a type of Neuro-Fuzzy network which has the fuzzy rules embedded within the neural network.

\section{Performance Metrics}

We measure the performance of the classifiers with respect to different performance metrics like accuracy, precision, recall, F-measure, area under ROC curve, and gamma statistic. More details of those measures could be found elsewhere [14,17,18].

The mean absolute error $E_{i}$ of an individual program $i$ is evaluated by the Equation (1):

$$
E_{i}=\frac{1}{n} \sum_{j=1}^{n}\left|P_{(i j)}-T_{j}\right|
$$

where $P_{(i j)}$ is the value predicted by the individual program $i$ for sample case $j$ (out of $n$ sample cases); and $T_{j}$ is the target value for sample case $j$. For a perfect fit, $P_{(i j)}=T_{j}$ and $E_{i}=0$. So, the $E_{i}$ index ranges from 0 to infinity, with 0 corresponding to the ideal.

The relative absolute error is also relative to a simple predictor, which is just the average of the actual values. In this case, though, the error is just the total absolute error instead of the total squared error. Thus, the relative absolute error takes the total absolute error and normalizes it by dividing by the total absolute error of the simple predictor.

Mathematically, the relative absolute error $E_{i}$ of an individual program $i$ is evaluated by the Equation (2):

$$
E_{i}=\sum_{j=1}^{n}\left|P_{(i j)}-T_{j}\right| / \sum_{j=1}^{n}\left|T_{j}-\bar{T}\right|
$$

where $P_{(i j)}$ is the value predicted by the individual program $i$ for sample case $j$ (out of $n$ sample cases); $T_{j}$ is the target value for sample case $j$; and $\bar{T}$ is given by the formula (Equation (3)):

$$
\bar{T}=\frac{1}{n} \sum_{j=1}^{n} T_{j}
$$

We also calculate the Root Mean Squared Error which is the square root of Equation (3) and Root Relative Squared Error is square root of Equation (4). 


\section{Survey Result}

To measure and investigate the performance on the selected classification methods namely Multilayer Perceptron (MLP) Neural Network, Bayes Network Classifier, J48graft (C4.5 Decision Tree Revision 8), JRip (RIPPER), Fuzzy Lattice Reasoning (FLR) classifier, we use the experiment procedures by WEKA whereas MLP, Naïve Bayes, C4.5 Decision Tree provided by TANAGRA and lastly Fuzzy Inference System (FIS), Adaptive Neuro-Fuzzy Inference System (ANFIS) experiment procedure provided by MATLAB. The $66 \%$ data is used for training and the remaining is for testing purposes that is shown in Table 2.

In this study, all data is considered as instances and features in the data are known as attributes. The simulation results are partitioned into several sub items for easier analysis and evaluation. Different performance matrix like TP rate, FP rate, and Precision, Recall, F-measure and $\mathrm{ROC}$ area are presented in numeric value during training and testing phase. The summary of those results by running the techniques in WEKA is reported in Table 3. Then we run the algorithms in Tanagra and results are reported through Tables 4 and 5. Performance measurement in Matlab environment is reported in Table 6. Tables 7 and 8 report different types of error measurement like mean absolute error and root mean squared error, the time taken to build model in second and Kappa statistic. Table 9 reports accurate and error rate that is represented in percentage value. Finally, Table 10 shows the rules that used FIS and ANFIS for MATLAB.

Table 2. Number of instances in the training and test data set.

\begin{tabular}{cccc}
\hline Data set & $\begin{array}{c}\text { No. of training } \\
\text { data }\end{array}$ & $\begin{array}{c}\text { No. of test } \\
\text { data }\end{array}$ & Total \\
\hline $\begin{array}{c}\text { Pima Indians } \\
\text { Diabetes }\end{array}$ & 507 & 261 & 768 \\
\hline
\end{tabular}

Table 3. Different performrance metrics running in WEKA.

\begin{tabular}{cccccccc}
\hline Classifier & Phase & TP rate & FP rate & Precision & Recall & F-measure & ROC Area \\
\hline \multirow{2}{*}{ MLP } & Train & 0.806 & 0.191 & 0.819 & 0.806 & 0.809 & 0.872 \\
& Test & 0.778 & 0.306 & 0.774 & 0.778 & 0.776 & 0.813 \\
\multirow{3}{*}{ BayesNet } & Train & 0.783 & 0.26 & 0.783 & 0.783 & 0.783 & 0.851 \\
& Test & 0.797 & 0.253 & 0.799 & 0.797 & 0.798 & 0.848 \\
\multirow{2}{*}{ J48graft } & Train & 0.841 & 0.241 & 0.842 & 0.841 & 0.836 & 0.888 \\
& Test & 0.785 & 0.189 & 0.816 & 0.785 & 0.792 & 0.803 \\
JRip & Train & 0.794 & 0.257 & 0.792 & 0.794 & 0.793 & 0.785 \\
& Test & 0.824 & 0.294 & 0.821 & 0.824 & 0.816 & 0.766 \\
FLR & Train & 0.358 & 0.344 & 0.774 & 0.358 & 0.2 & 0.507 \\
& Test & 0.67 & 0.662 & 0.582 & 0.67 & 0.572 & 0.504 \\
\hline
\end{tabular}

Table 4. Different performance metrics in TANAGRA.

\begin{tabular}{ccc}
\hline Classifier & Recall & Precision \\
\hline MLP & 0.8275 & 0.8275 \\
Naïve bayes & 1 & 1 \\
C4.5 & 0.90465 & 0.90465 \\
\hline
\end{tabular}

Table 5. Performance measuring in training and test data set using TANAGRA.

\begin{tabular}{cccc}
\hline Classifier & Accuracy & Error rate & Time (seconds) \\
\hline MLP & $83.85 \%$ & $16.15 \%$ & 2.36 \\
Naïve bayes & $100.00 \%$ & $0.00 \%$ & 0.001 \\
C4.5 & $90.63 \%$ & $9.38 \%$ & 0.031 \\
\hline
\end{tabular}

Table 6. Performance measuring in rule based fuzzy approach using MATLAB.

\begin{tabular}{ccccc}
\hline Learning systems & Training/test epochs & $\begin{array}{c}\text { Avg. error after } \\
\text { training/test }\end{array}$ & $\begin{array}{c}\text { No. of } \\
\text { extracted rules }\end{array}$ & Rules accuracy (\%) \\
\hline $\begin{array}{c}\text { Fuzzy Inference System } \\
\text { Adaptive Neuro-Fuzzy } \\
\text { Inference System }\end{array}$ & 500 & 7.6358 & 7 & 71.51 \\
\hline
\end{tabular}


Table 7. Error measurement for different classifiers in WEKA.

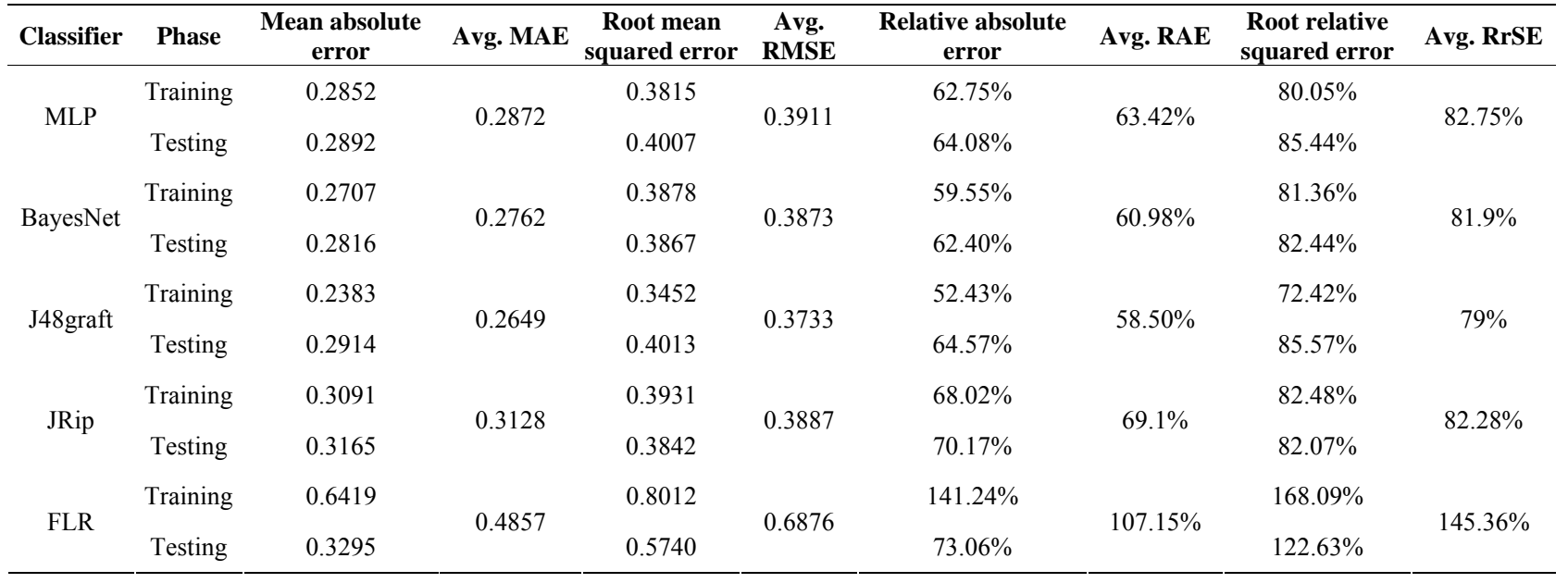

Table 8. Performance measuring in training and test data set using WEKA.

\begin{tabular}{|c|c|c|c|c|c|c|c|c|c|}
\hline Classifier & Phase & Accuracy & Avg. AC & Error Rate & Avg. ER & Time (seconds) & Avg. TT & Kappa statistic & Avg. KS \\
\hline \multirow{2}{*}{ MLP } & Training & $80.60 \%$ & \multirow{2}{*}{$79.19 \%$} & $19.40 \%$ & \multirow{2}{*}{$20.81 \%$} & 101.08 & \multirow{2}{*}{63.13} & 0.5904 & \multirow{2}{*}{0.5358} \\
\hline & Testing & $77.78 \%$ & & $22.22 \%$ & & 25.17 & & 0.4812 & \\
\hline \multirow{2}{*}{ BayesNet } & Training & $78.26 \%$ & \multirow{2}{*}{$78.98 \%$} & $21.74 \%$ & \multirow{2}{*}{$21.03 \%$} & 0.03 & \multirow{2}{*}{0.04} & 0.5218 & \multirow{2}{*}{0.5305} \\
\hline & Testing & $79.69 \%$ & & $20.31 \%$ & & 0.05 & & 0.5391 & \\
\hline \multirow[b]{2}{*}{ J48graft } & Training & $84.11 \%$ & \multirow[b]{2}{*}{$81.33 \%$} & $15.89 \%$ & \multirow[b]{2}{*}{$18.68 \%$} & 0.19 & \multirow[b]{2}{*}{0.135} & 0.6319 & \multirow[b]{2}{*}{0.59} \\
\hline & Testing & $78.54 \%$ & & $21.46 \%$ & & 0.08 & & 0.5481 & \\
\hline \multirow{2}{*}{ JRip } & Training & $79.43 \%$ & \multirow{2}{*}{$80.91 \%$} & $20.57 \%$ & \multirow{2}{*}{$19.10 \%$} & 0.3 & \multirow{2}{*}{0.29} & 0.5425 & \multirow{2}{*}{0.5542} \\
\hline & Testing & $82.38 \%$ & & $17.62 \%$ & & 0.28 & & 0.5658 & \\
\hline \multirow{2}{*}{ FLR } & Training & $35.81 \%$ & \multirow{2}{*}{$51.43 \%$} & $64.19 \%$ & \multirow{2}{*}{$48.57 \%$} & 0.03 & \multirow{2}{*}{0.025} & 0.0098 & \multirow{2}{*}{0.0107} \\
\hline & Testing & $67.05 \%$ & & $32.95 \%$ & & 0.02 & & 0.0115 & \\
\hline
\end{tabular}

Table 9. Accuracy in percentage for different classification comparing three tools.

\begin{tabular}{|c|c|c|c|c|c|c|c|c|}
\hline Tool & MLP & BayesNet/Naïve bayes & C4.5/J48graft & JRip & FLR & FIS & ANFIS & Average \\
\hline WEKA & $79.19 \%$ & $78.98 \%$ & $81.33 \%$ & $80.91 \%$ & $51.43 \%$ & nill & nill & $74.37 \%$ \\
\hline TANAGRA & $83.85 \%$ & $100 \%$ & $90.63 \%$ & nill & nill & nill & nill & $91.49 \%$ \\
\hline MATLAB & nill & nill & nill & nill & nill & $71.51 \%$ & $78.79 \%$ & $75.15 \%$ \\
\hline
\end{tabular}

Table 10. Sample rules framed for the proposed FIS and ANFIS.

\begin{tabular}{|c|c|c|c|c|c|c|c|c|c|c|}
\hline \multirow[b]{2}{*}{ Rule No. } & \multicolumn{8}{|c|}{ IF } & \multicolumn{2}{|c|}{ THEN } \\
\hline & preg. & plas & bp & skin & insl & bmi & $\mathrm{dpf}$ & age & class 0 (weight) & class 1 (weight) \\
\hline 1 & 0 & $\leq 103$ & $>40$ & $\leq 26$ & $\leq 156$ & $\leq 35.3$ & $\leq 0.179$ & $\leq 34$ & 0.955 & 0.5 \\
\hline 2 & $\leq 3$ & not define & not define & $\leq 35$ & $>156$ & $\leq 35.3$ & $\leq 0.787$ & not define & 0.5 & 0.928 \\
\hline 3 & not define & not define & not define & not define & not define & not define & $\leq 0.179$ & $\leq 34$ & 0.955 & 0.5 \\
\hline 4 & not define & $\leq 103$ & not define & not define & not define & not define & $\leq 0.787$ & not define & 0.944 & 0.5 \\
\hline 5 & not define & not define & not define & not define & $\leq 156$ & $\leq 35.3$ & not define & $>34$ or $\leq 37$ & 0.912 & 0.5 \\
\hline 6 & not define & $>135$ & not define & not define & $\leq 185$ & $>33.7$ & $\leq 1.096$ & $>37$ & 0.5 & 0.928 \\
\hline 7 & 6 & $>103$ & not define & not define & not define & $>35.3$ & $\leq 1.096$ & $>34$ & 0.5 & 0.909 \\
\hline
\end{tabular}




\section{Result Analysis and Discussion}

In this study, we examine the performance of different classification methods that could generate accuracy and some error to diagnosis the data set.

According to Figure $\mathbf{2}$ and Table 8, we can clearly see the highest accuracy is $81.33 \%$ belongs to J48graft and lowest accuracy is $51.43 \%$ that belongs to FLR. The total time required to build the model is also a crucial parameter in comparing the classification algorithm.

Based on Figure 3 and Table 7, we can compare errors among different classifiers in WEKA. We clearly find out that J48graft is the best, second best is the Bayes Net and MLP \& JRip is moderate but FLR is arguable. An algorithm which has a lower error rate will be preferred as it has more powerful classification capability and ability in terms of medical and bioinformatics fields.

From Figure 4, we see that FLR classifier requires the shortest time which is around 0.025 seconds compared to other. MLP algorithm requires the longest model building time which is around 63.13 seconds. The second one the list is Bayes network with 0.04 seconds, whereas J48graft takes 0.135 seconds.

Kappa statistic is used to assess the accuracy of any particular measuring cases, it is usual to distinguish between the reliability of the data collected and their validity [18]. The average Kappa score from the selected al-

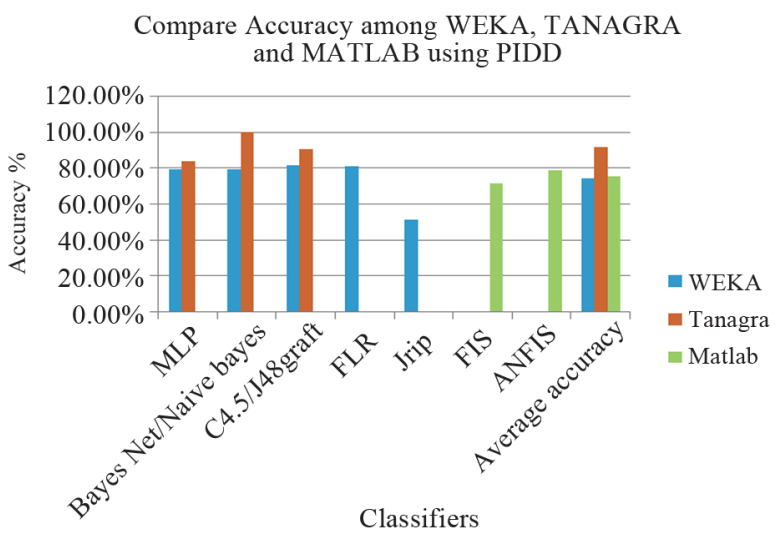

Figure 2. Accuracy of three tools using PIDD.

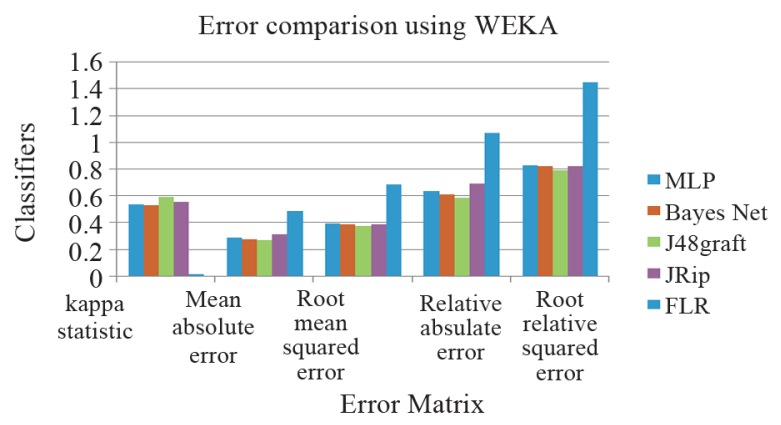

Figure 3. Error comparison for WEKA.

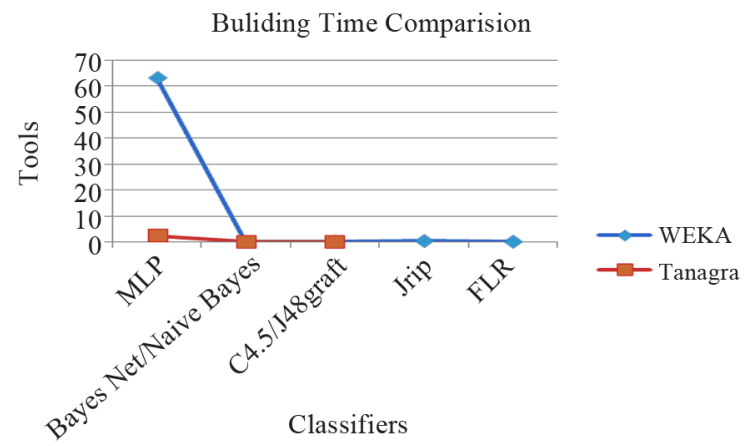

Figure 4. Time comparison of WEKA \& TANAGRA

gorithm is around $0.01-0.59$. Based on the Kappa Statistic criteria, the accuracy of this classification purposes is substantial [14]. So according to best average kappa statistic the J48graft classifier is best among others. In Table 5 investigated the accuracy using TANAGRA tools for three classifiers like MLP, Naïve Bayes and C4.5. According to the TANAGRA and algorithms, Naïve Bayes is best comparatively others classifiers cause $100 \%$ accuracy achieved by Naïve Bayes and take time to build model is $0.001 \mathrm{~s}$ that also lowest time compare to others.

Cluster analysis is a way to examine similarities and dissimilarities of observations or objects. Data often fall naturally into groups, or clusters, of observations, where the characteristics of objects in the same cluster are similar and the characteristics of objects in different clusters are dissimilar. In this examination, cluster analysis is used for the purpose of segregating the patients with high risk and low risk. Grouping of clusters are used to identify the patients who need the emergency care. Using the MLP, BayesNet, JRip, FLR or J48graft in WEKA using EM cluster are shaped by training them with input/output data rather than specifying them automatically. Figure 5 shows the WEKA cluster analysis.

Another figure generated from MATLAB result is shown in Table 7. Since same iteration and same dataset used, average error is similar. But rules accuracy is $71.51 \%$ and $78.79 \%$ of FIS and ANFIS respectively for different network architecture. Fuzzy Inference System (FIS) and Adaptive Neuro-Fuzzy Inference Systems (ANFIS) classifiers are chosen from MATLAB fuzzy logic toolbox. IF-THEN rules are used in adaptive classifiers. For performance measurement, we use 7 IF-THEN fuzzy rules and mamdani membership for FIS and sugeno for ANFIS. Figure 6 shows the FIS model with mamdani membership function.

Figure 7 shows the membership function for input and output variable. Eight attributes need different membership function according to rules that showed in Table 10. All membership functions are continuous, all membership functions map an interval [a, b] to $[0,1], \mu[a, b]$ to 


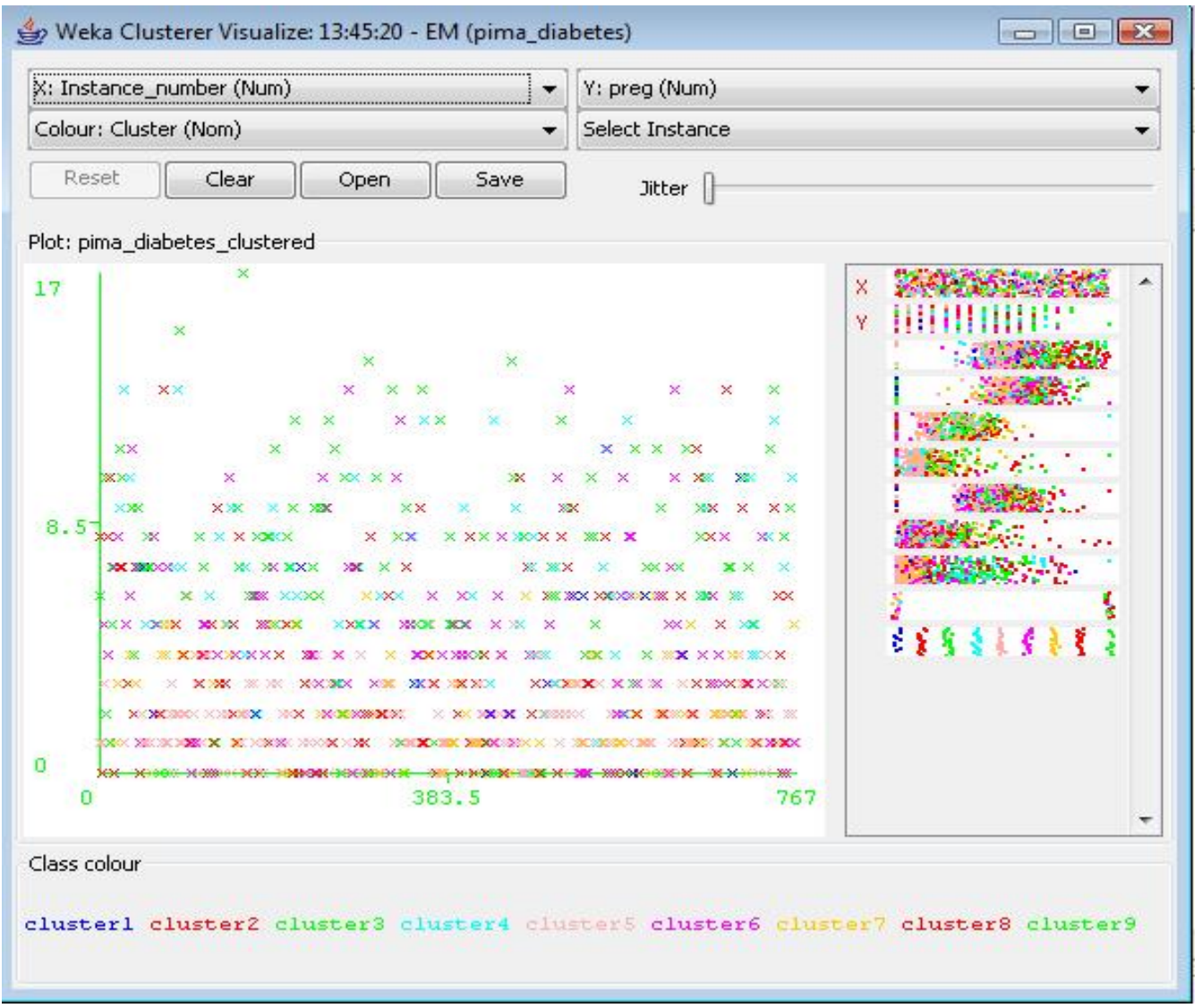

Figure 5. Generation of cluster for WEKA using PIDD.

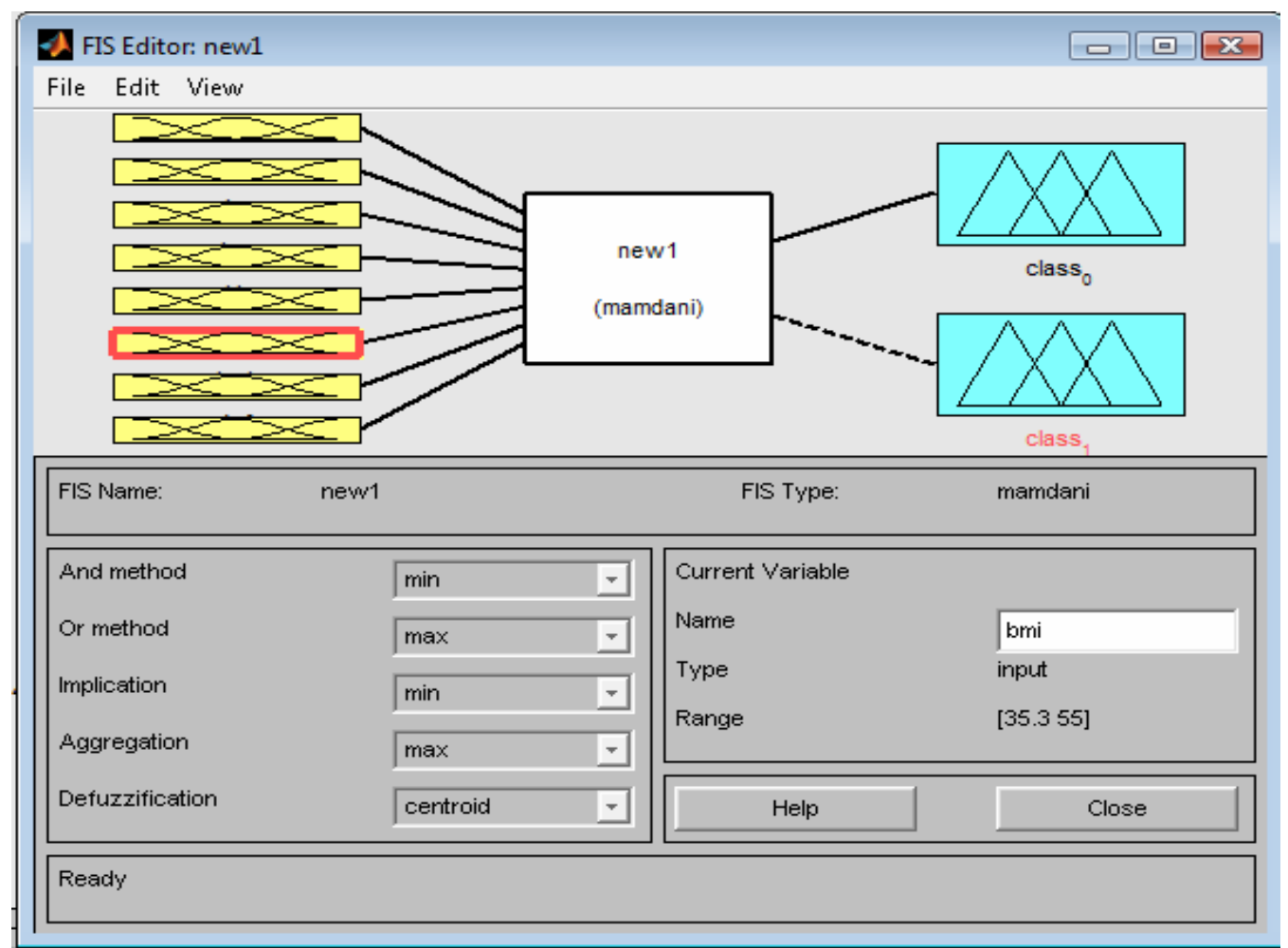

Figure 6. Fuzzy Inference System. 

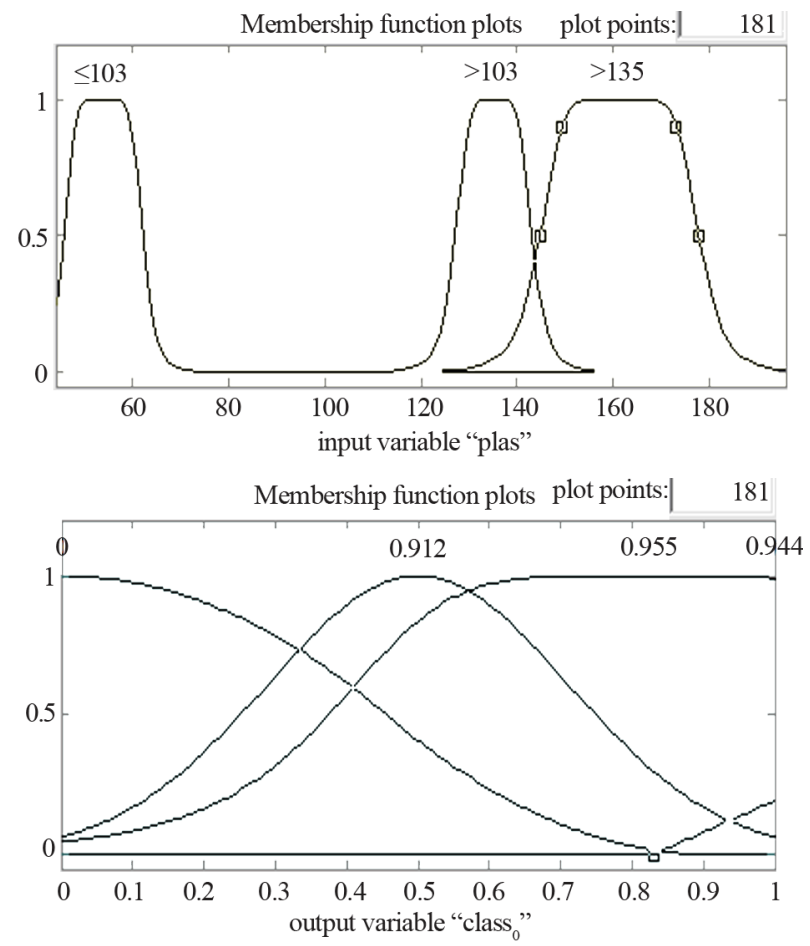

Figure 7. Membership function of input and output.

$[0,1]$. Figure 7 illustrates plasma attribute that models gbellmf function (Generalized bell-shaped-in membership function) for condition. The generalized bell function depends on three parameters $a, b$, and $c$ as given by

$$
f(x ; a, b, c)=\frac{1}{1+\left|\frac{x-c}{a}\right|^{2 b}}
$$

where the parameter $b$ is usually positive. The parameter $c$ locates the center of the curve. We have to call the function "gbellmf" with the parameter vector params whose entries are $a, b$, and $c$, respectively. The syntax of gbellmf is $y=\operatorname{gbellmf}(x$, params $)$.

Example:

$$
\begin{aligned}
& \mu_{\text {positive }}(x)= \begin{cases}1, & \text { if } x \leq 103 \\
\frac{1}{1+\left(\frac{x-103}{a}\right)^{2}}, & \text { if } x>103\end{cases} \\
& \mu_{\text {negative }}(x)= \begin{cases}0, & \text { if } x \leq 135 \\
\frac{1}{1+\left(\frac{x-135}{a}\right)^{-2}}, & \text { if } x>135\end{cases}
\end{aligned}
$$

The second part of Figure 7 illustrates the class 0 output of plasma input, membership function according to diabetes condition or rules. For class output four membership functions are used. For example, zmf (Z-shaped built-in membership function), gaussmf (Gaussian curve built-in membership function), gbellmf (Generalized bell-shaped-in membership function) and trimf (Triangular-shaped built-in membership function) are used. 0, 0.912, 0.955 and 0.944 plasma output for $\mathrm{zmf}$, gaussmf, gbellmf and trimf membership function respectively.

The rule base consists of a set of fuzzy propositions and is derived from the knowledge base of the medical expertise. A fuzzy proposition or a statement establishes a relationship between different input fuzzy sets and output fuzzy sets. In this phase, the decision rules are constructed for input parameter and control output values to find the positive or negative diabetes. In order to validate the fuzzy logic approach used in construction of Fuzzy Inference System, the extensive simulation is carried out on the simulated model.

The system responses with variations defined in the membership functions as a rule viewer, surface viewer. Data training, checking, testing with sample data is done to capture the error by ANFIS using FIS model. ANFIS uses hybrid learning rules, which combines the gradient method and the least squares estimate to identify parameters. The ANFIS network structure used in this research is depicted in Figure 8.

The rule-base constructed are simulated using MATLAB to identify the output parameter class 0 or class 1 that means tested positive and negative. Figures $\mathbf{9}$ and $\mathbf{1 0}$ finally report the simulation view of Fuzzy Inference System and Adaptive Neuro-Fuzzy Inference System (ANFIS). In ANFIS Editor, membership functions are shaped by training them with input/output data rather than specifying them manually. The training algorithm is a combination (hybrid) with a least square system to learn from the data.

To measure the diagnosis accuracy with Fuzzy approach, training and testing iteration is 500 epochs (for all tools and all classifiers). By ANFIS we achieve $78.79 \%$ accuracy that's highest than FIS (71.51\%). Table 10 reports the diabetes diagnosis rules.

Finally, Figures 11 and $\mathbf{1 2}$ show the surface construction of negative and positive class.

\section{Conclusion and Future Work}

The objective of this study is to evaluate and investigate nine selected classification algorithms based on WEKA, TANAGRA and MATLAB. The best algorithm in WEKA is J48graft classifier with an accuracy of $81.33 \%$ that takes 0.135 seconds for training. In TANAGRA, Naïve Bayes classifier provides accuracy of $100 \%$ with training time 0.001 seconds. In MATLAB, ANFIS has $78.79 \%$ accuracy If we compare with average accuracy, TANAGRA machine learning tool is the best compared to WEKA and MATLAB. Those results suggest that among the machine learning algorithm tested on PIDD, 


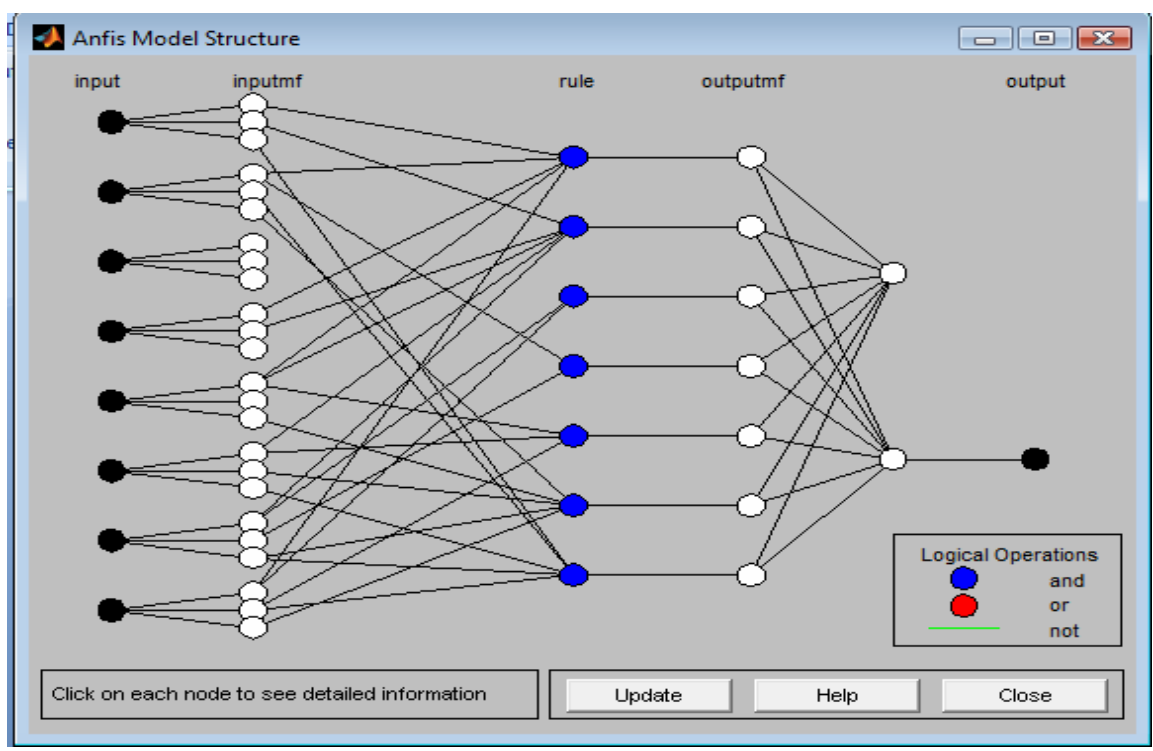

Figure 8. ANFIS network structure.

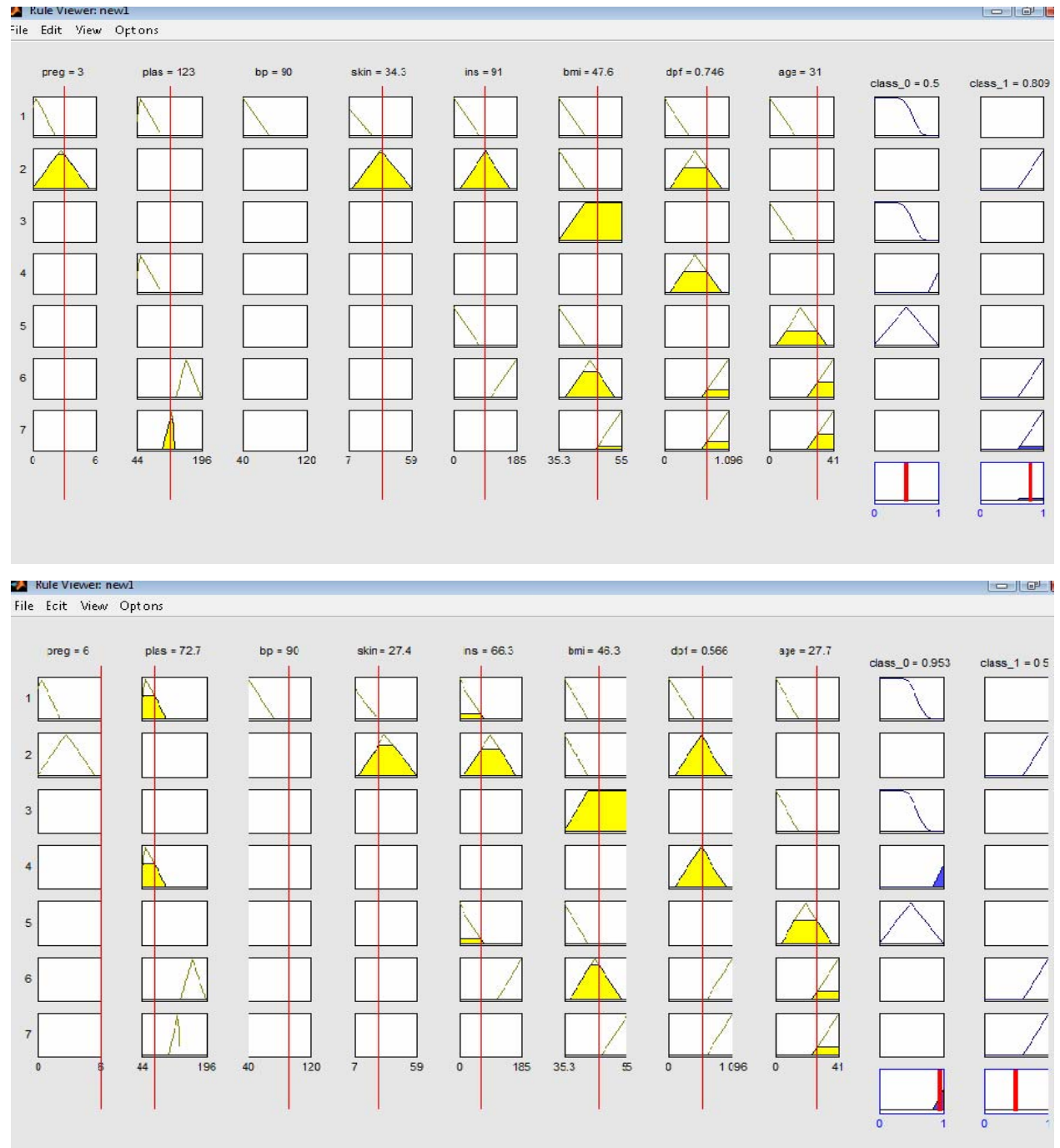

Figure 9. Simulation view of rules base of diabetes using FIS. 

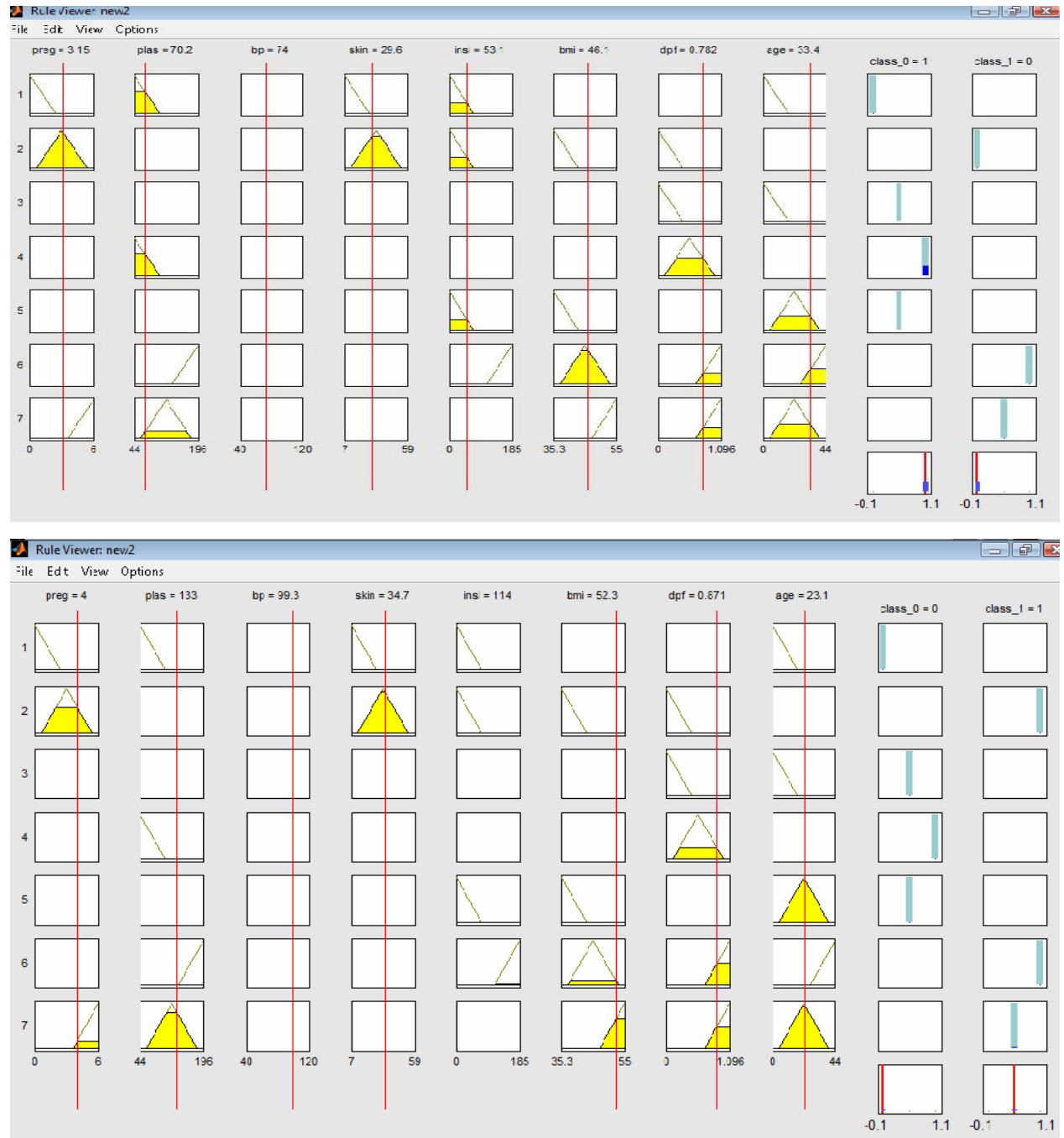

Figure 10. Simulation view of rule base of diabetes using ANFIS.

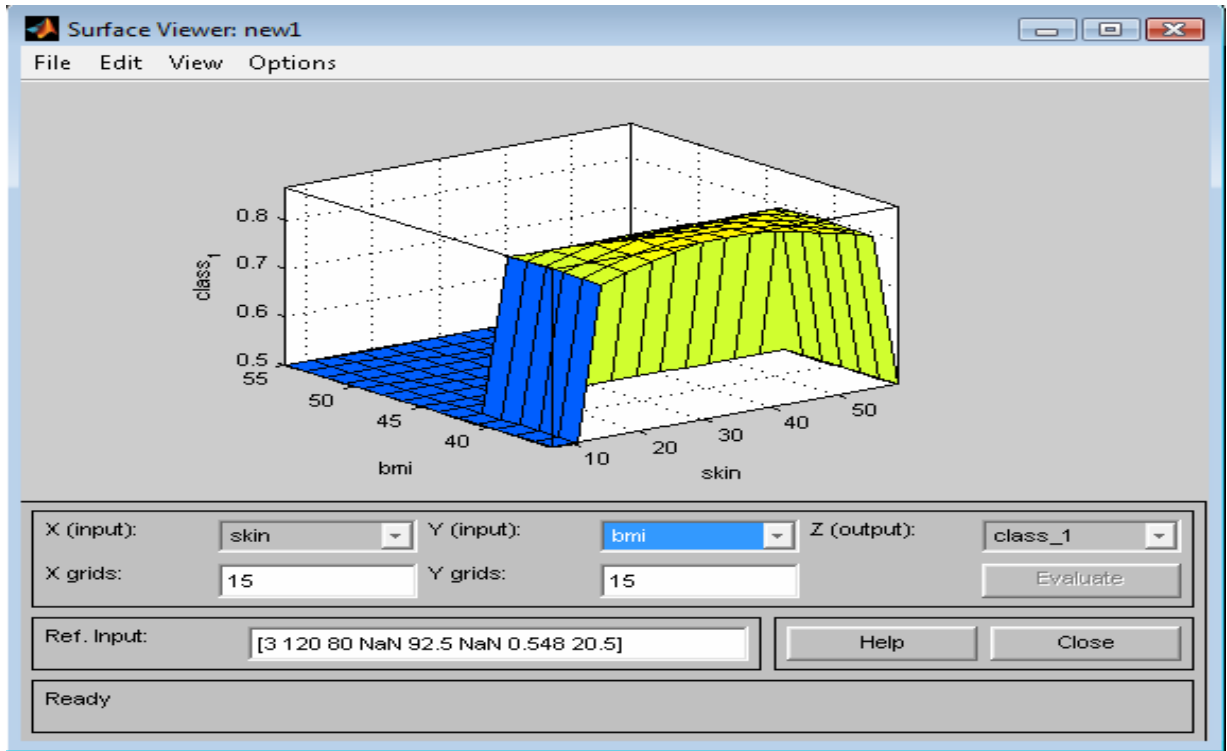

Figure 11. Surface construction of FIS and ANFIS with skin vs. bmi. 


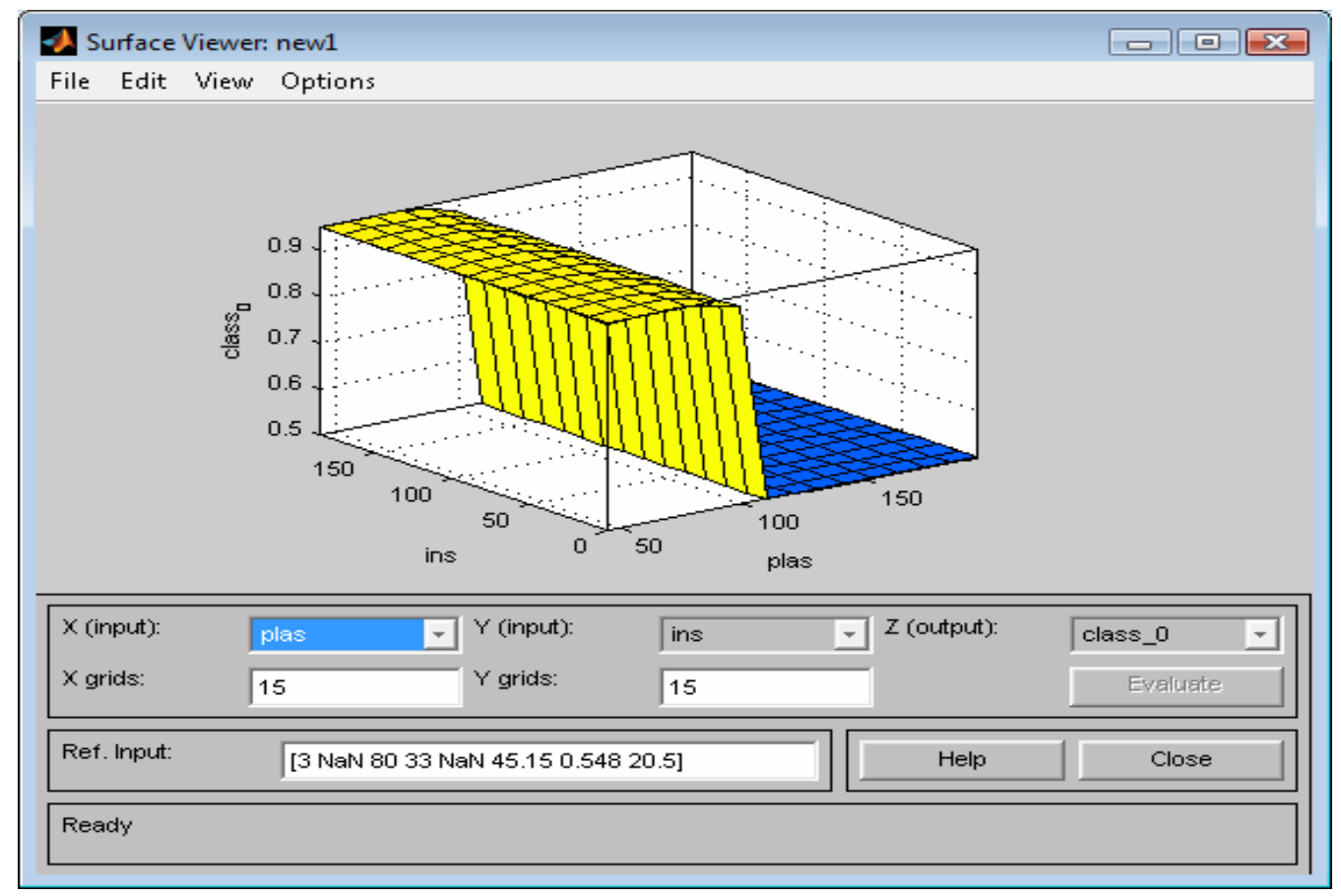

Figure 12. Surface construction of FIS and ANFIS with plas vs. ins.

Bayes network classifier like NaiveBayes, Bayes-Net and Tree pruning classifier like J48graft and Rule learner like Adaptive Neuro-Fuzzy Inference System has the potential to significantly improve over the conventional classification methods for use in medical or in general, bioinformatics field. However there is chance of improvement. First, the misclassification cost is not considered explicitly in this research. In future, cost-sensitive learning might make the study more practical and valuable. Second, in this survey we use only 7 rules for FIS and ANFIS but if increasing the rules we could get more accurate diagnosis result.

\section{REFERENCES}

[1] N. J. Nilsson, "Introduction to Machine Learning," 2010. http://ai.stanford.edu/ nilsson/mlbook.html

[2] M. S. Sapna and D. A. Tamilarasi, "Fuzzy Relational Equation in Preventing Neuropathy Diabetic," International Journal of Recent Trends in Engineering, Vol. 2, No. 4, 2009, p. 126.

[3] L. Carnimeo and A. Giaquinto, "An Intelligent System for Improving Detection of Diabetic Symptoms in Retinal Images," IEEE International Conference on Information Technology in Biomedicine, Ioannina, 26-28 October 2006.

[4] R. Radha and S. P. Rajagopalan, "Fuzzy Logic Approach for Diagnosis of Diabetes," Information Technology Journal, Vol. 6, No. 1, pp. 96-102. doi:10.3923/itj.2007.96.102

[5] P. Jeatrakul and K. W. Wong, "Comparing the Performance of Different Neural Networks for Binary Classifica- tion Problems," The 8th International Symposium on Natural Language Processing, Bangkok, 20-22 October 2009, pp. 111-115. doi:10.1109/SNLP.2009.5340935

[6] Q. Q. Zhou, M. Purvis and N. Kasabov, "Membership Function Selection Method for Fuzzy Neural Networks," University of Otago, Dunedin, 2007. http://otago.ourarchive.ac.nz/handle/10523/1027

[7] T.-H. Lin and V.-W. Soo, "Pruning Fuzzy ARTMAP Using the Minimum Description Length Principle in Learning from Clinical Databases," Proceedings of the 9th International Conference on Tools with Artificial Intelligence, Newport Beach, 3-8 November 1997, pp. 396-403.

[8] F. Ensan, M. H. Yaghmaee and E. Bagheri, "Fact: A New Fuzzy Adaptive Clustering Technique," The 11th IEEE Symposium on Computers and Communications, Sardinia, 26-29 June 2006, pp. 442-447. doi:10.1109/ISCC.2006.73

[9] UCI Machine Learning Repository. http://www.ics.uci.edu/mlearn/MLRepository.html

[10] S. W. Purnami, A. Embong, J. M. Zain and S. P. Rahayu, "A New Smooth Support Vector Machine and Its Applications in Diabetes Disease Diagnosis," Journal of Computer Science, Vol. 5, No. 12, pp. 1006-1011.

[11] P. Werbos, "Beyond Regression: New Tools for Prediction and Analysis in the Behavioral Sciences," Ph.D. Thesis, Harvard University, Cambridge, 1974.

[12] G. H. John and P. Langley, "Estimating Continuous Distributions in Bayesian Classifiers," Proceedings of the 11th Conference on Uncertainty in Artificial Intelligence, San Francisco, 1995, pp. 338-345.

[13] J. Quinlan, “C4.5: Programs for Machine Learning," Morgan Kaufmann, San Mateo, 1993. 
[14] I. H. Witten and E. Frank, "Data Mining: Practical Machine Learning Tools and Techniques," 2nd Edition, Morgan Kaufmann, San Francisco, 2005.

[15] The Mathworks-Fuzzy Logic Toolbox, 2006. http://www.mathworks.ch/access/helpdeskr13/help/toolb ox/fuzzy/fuzzy.html

[16] Jang and J.-S. Roger, "Anfis: Adaptive-Network-Based Fuzzy Inference System,” IEEE Transactions on Systems,
Man, and Cybernetics, Vol. 23, No. 3, 1993, pp. 665-685. doi:10.1109/21.256541

[17] J. W. Han and M. Kanber, "Data Mining Concept and Techniques," Morgan Kaufmann Publishers, Burlington, 2000.

[18] Kappa Statistic.

http://www.dmi.columbia.edu/homepages/chuangj/kappa 